\title{
Ab-initio Calculation of the Properties of Permalloys Used in Electrical Machines
}

\author{
Marco Trapanese, member IEEE \\ Department of Electrical Engineering, Palermo University \\ Viale delle Scienze ed.9, 90128 Palermo, Italy \\ mtrap@diepa.unipa.it
}

\begin{abstract}
In this paper an ab-initio calculation that allows to describe the main features of permalloys is presented. The calculation is based on Korringa-Kohn-Rostoker electronic structure method. The dependence of the magnetocrystalline anisotropy and of the magnetostriction on the concentration of non magnetic addiction is computed.
\end{abstract}

\section{INTRODUCTION}

The use of very soft material in electrical machines permits to enhance the efficiency of the machines. The prediction of the properties of the used materials is extremely useful in order to optimize the design of the machine. In this paper an ab-initio calculation of the properties of permalloys is presented. Permalloys are among the softest materials used in electrical machine. The calculation is based on the Korringa-KohnRostoker electronic structure method. The parameters, that are calculated, are the magnetocrystalline anisotropy and the magnetostriction. Their dependence on the concentration of the non magnetic addiction of the alloy is computed.

\section{MAGNETOSTRICTION AND MAGNETIC ANISOTROPY OF METALS AND ALLOYS}

The softness of a magnetic material is caused by a low value of both the magnetic anisotropy constant and the magnetostriction coefficient. The softest among permalloys is $\mathrm{Ni}_{80} \mathrm{Fe}_{20}$, moreover it has been shown that the addiction of small amount of non magnetic elements in this alloy can result in an improvement of softness. In this paper the Magnetic Anisotropy Energy (MAE) and the magnetostriction is calculated and it is shown that this calculation can explain the two facts above said.

Magnetic anisotropy energy can be computed as follows:

$$
\Delta F\left(e_{1}, e_{e}\right)=\int_{f}^{E_{f}^{1}} \varepsilon n_{e_{1}}(\varepsilon) d \varepsilon-\int_{f}^{E_{f}^{2}} \varepsilon n_{e_{2}}(\varepsilon) d \varepsilon
$$

where $E_{F}^{1}$ and $E_{F}^{2}$ are the Fermi energies when the system is magnetized along the direction $e_{1}$ and $e_{2}$ and $n_{e x}$ are the electronic densities of states. In order to calculate the density of states the algorithm proposed in [1] has been followed. The magnetostriction constant is the change of length of the elementary cell when an external field is applied and can be computed from the rate of change of MAE.

The magnetic anisotropy energy and the magnetostriction constant have been computed in the case of a $\mathrm{Ni}_{80} \mathrm{Fe}_{20}$ and in the case of $\mathrm{Ni}_{75} \mathrm{FE}_{25}$. The results are shown in fig. 1 .

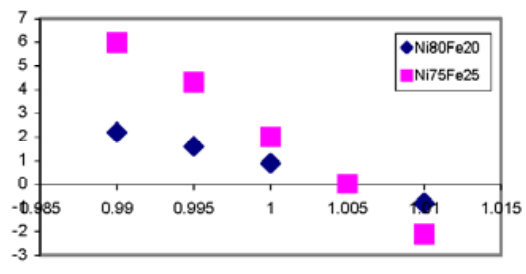

Fig.1 The magnetic anisotropy energy $([001]-[111]$, the easy axis is [111]) in mev vs $\mathrm{c} / \mathrm{a}$ (c/a is the tetragonal variation) in two different composition of permalloy

From fig. 1 it can be seen how the softest alloy, that corresponds to the lowest MAE, is $\mathrm{Ni}_{80} \mathrm{Fe}_{20}$. However, it must be said that the experimental MAE is higher than the computed one.

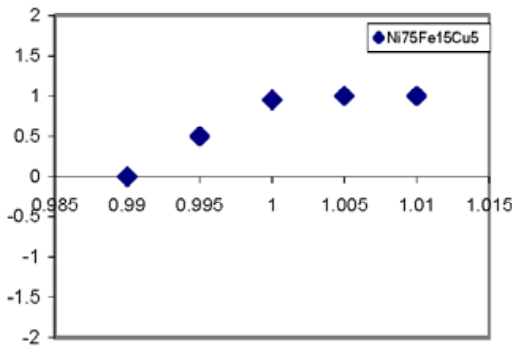

Fig. 2 The magnetic anisotropy energy([001]-[111], the easy axis is [111]) in mev vs $\mathrm{c} / \mathrm{a}$ when a small quantity of $\mathrm{Cu}$ is added.

From fig.2, it can be seen how the addiction of a small quantity of $\mathrm{Cu}$ can reduce the magnetic anisotropy energy and therefore enhances the softness of the material.

\section{CONCLUSIONS}

Korringa-Kohn-Rostoker electronics structure method has been used for the calculation of magnetic anisotropy energy and the magnetostriction coefficient. It has allowed to qualitatively describe the enhancement of softness of the $\mathrm{Ni}_{80} \mathrm{Fe}_{20}$ alloy when a small quantity of $\mathrm{Cu}(0.05 \%)$ is added.

It is under investigation the application of the method in order to reach a quantitative description of the magnetic properties of the material used in electric machines.

\section{REFERENCES}

[1] E.Bruno and B.Ginatempo, "Algorithms for Korringa-Kohn-Rostoker electronic structure calculation in any Bravais lattice", Physical Review B, Vol. 55, N.19, pp. 12946-12955, May 1997. 